\title{
Editorial
}

\section{Hypertrophic cardiomyopathy: is there a role for amiodarone?}

The usual mechanism of sudden death in various forms of heart disease is thought to be ventricular arrhythmia. ${ }^{1-3}$ However, antiarrhythmic drugs have not been shown conclusively to prevent sudden death, except in the case of $\beta$ blockers, which improve survival in patients with heart failure and after myocardial infarction (MI), ${ }^{4}$ although this may or may not relate to their antiarrhythmic properties. Indeed, class I antiarrhythmic agents and sotalol have been reported to increase mortality because of their proarrhythmic properties. ${ }^{56}$ Amiodarone is attractive because it is a potent antiarrhythmic that does not have significant negative inotropic properties, ${ }^{7}$ and has fewer proarrhythmic properties than class I agents. ${ }^{7}$ Studies in patients with heart failure have demonstrated conflicting results indicating that there may be a survival benefit with amiodarone in non-ischaemic but not ischaemic heart failure. ${ }^{89}$ In post-MI patients, reductions in arrhythmic death, but not total mortality, have been reported in the CAMIAT and EMIAT studies. ${ }^{10} 11$

\section{Potential role for amiodarone in hypertrophic cardiomyopathy}

Although the major incidence of sudden death occurs in older people, sudden death does occur in adolescents and young adults. ${ }^{212}$ Hypertrophic cardiomyopathy is a common cause of sudden death in these younger pateints ${ }^{13}$ and prevention of this complication is a major challenge. ${ }^{12}$ Risk factors for sudden death in patients with hypertrophic cardiomyopathy include family history of sudden death (especially if in more than one family member), recurrent syncope, abnormal exercise blood pressure response (hypotension or failure of blood pressure to rise), and episodes of non-sustained ventricular tachycardia on Holter monitoring. ${ }^{14}{ }^{15}$ The last association, reported independently by two groups in the early $1980 \mathrm{~s},{ }^{16}{ }^{17}$ led to the hypothesis that amiodarone might reduce sudden death in high risk individuals with hypertrophic cardiomyopathy. In 1985 McKenna et al reported their experience with amiodarone treatment of high risk patients with hypertrophic cardiomyopathy. ${ }^{18}$ This was a non-randomised but well controlled study in which a later high risk cohort (defined as non-sustained ventricular tachycardia on ambulatory ECG monitoring) treated with amiodarone was compared with an earlier high risk cohort receiving a class I agent, usually disopyramide with or without a $\beta$ blocker. The earlier cohort had a $7 \%$ annual mortality, but there were no deaths in the amiodarone treated group over a mean follow up period of 2.6 years.

The study has several limitations. First, it was not randomised or placebo controlled but a comparison of

\author{
Glossary \\ CAMIAT: Canadian Amiodarone Myocardial \\ Infarction Arrhythmia Trial \\ EMIAT: European Myocardial Infarct Amiodarone \\ Trial \\ GESICA: Grupo de Estudio de la Sobrevida en la \\ Insuficienca Cardiaca en Argentina
}

consecutive, well matched patient groups. Second, the earlier cohort had received agents that we now believe may have increased the risk of sudden death. Third, the study was small, and absolute event rates were low. Nevertheless, it offered some evidence that amiodarone might reduce the incidence of sudden death in high risk patients with hypertrophic cardiomyopathy. On the basis of this study, amiodarone was used by a number of groups for the prevention of sudden death in patients with hypertrophic cardiomyopathy and non-sustained ventricular tachycardia on ambulatory ECG monitoring.

\section{Difficulties in identifying high risk individuals}

In addition to the of lack randomised trial data, several other factors have limited the widespread use of amiodarone in patients with hypertrophic cardiomyopathy, particularly given its relatively toxic side effect profile. First, the definition of high risk patients based on non-sustained ventricular tachycardia on Holter monitoring has certain drawbacks. In older adults, the absence of non-sustained ventricular tachycardia has a high negative predictive accuracy for sudden death, but in children and adolescents this is not the case ${ }^{19}$; thus, until recently, identification of high risk patients in this age group has been very difficult. Second, in an older adult population the absence of non-sustained ventricular tachycardia has a positive predictive accuracy of approximately $20 \%$ - that is, many patients will be treated unnecessarily using this criterion. Third, concerns have developed regarding the extrapolation of results from highly specialised tertiary referral centres to all patients with hypertrophic cardiomyopathy. ${ }^{20}{ }^{21}$ In Maron et al and McKenna et al's original series ${ }^{16}{ }^{17}$ the annual mortality rates for patients with non-sustained ventricular tachycardia (not treated with amiodarone) were $8 \%$ and $7 \%$, respectively. More recent data from non-tertiary referral centres suggests a lower risk of sudden death. ${ }^{22}$ Spirito et al found a sudden death rate of $1.4 \%$ per year in patients with non-sustained ventricular tachycardia compared with $0.9 \%$ in those without this arrhythmia. ${ }^{24}$ In most of these patients the episodes of non-sustained ventricular tachycardia were relatively brief or infrequent, and it has been argued that this may explain the lower event rate; however, this question remains unanswered.

\section{Is amiodarone proarrhythmic?}

There have been reports of sudden death in patients with hypertrophic cardiomyopathy despite amiodarone treatment. ${ }^{25-28}$ Fananapazir et al suggested that amiodarone may even increase the risk of sudden death as a result of its proarrhythmic effects. ${ }^{27}$ Using an aggressive programmed electrical stimulation protocol, induction of ventricular tachycardia was "easier" or occurred only with amiodarone in 18 of 35 patients studied. ${ }^{27}$ In another study from the same group, amiodarone was given to 50 patients for symptoms refractory to conventional treatment (21 of whom had ventricular tachycardia on Holter monitoring). ${ }^{26}$ Seven sudden deaths occurred during a mean follow up of 2.2 years, six within five months of initiation of treatment. The dosage of amiodarone used by Fananapazir's group was high $(1600 \mathrm{mg} /$ day loading and $400 \mathrm{mg}$ /day 
maintenance), and mean amiodarone blood concentrations were $>2 \mu \mathrm{g} / \mathrm{ml}$ - much higher than recommended by McKenna's group. In general, side effects of amiodarone appear to be dose related ${ }^{29}$ and the results of these two studies may represent proarrhythmia related to high dosage. The GESICA, CAMIAT, and EMIAT studies used lower doses of amiodarone and there was no evidence of major proarrhythmic effects. ${ }^{81011}$

In this issue, Cecchi et al report their experience in an unselected, non-tertiary population of 167 consecutive patients with hypertrophic cardiomyopathy. ${ }^{30}$ Ninety patients did not have non-sustained ventricular tachycardia on Holter monitoring, 38 had isolated, infrequent non-sustained ventricular tachycardia, and 39 had multiple-repetitive non-sustained ventricular tachycardia (similar to the prevalence noted by Maron's and McKenna's groups). Cecchi et al made the assumption in their management protocol that infrequent non-sustained ventricular tachycardia was relatively benign, but multiplerepetitive non-sustained ventricular tachycardia was malignant, and treated only patients with the latter with low dose (mean $220 \mathrm{mg} /$ day) amiodarone. As with McKenna et al's study, this was not randomised or placebo controlled. No significant differences in overall survival were seen between the three groups during follow up (mean 10 years). Only one sudden death occurred (in the isolated, infrequent non-sustained ventricular tachycardia group). Eight deaths occurred due to heart failure (four in the group without non-sustained ventricular tachycardia and four in the multiple-repetitive non-sustained ventricular tachycardia group). This study does not permit the definite conclusion that amiodarone prevents sudden death, but it is consistent with McKenna et al's overall beneficial experience and (at the dosage used) is at odds with Fanapazir et al's report of serious proarrhythmic effects. Furthermore, it confirms that in this non-tertiary referral population, the risk of sudden death in patients with isolated infrequent nonsustained ventricular tachycardia is low, and these patients may not need treatment with an agent that has a potentially toxic side effect profile.

\section{Is there a consensus?}

Thus, major problems remain in therapeutic decision making in hypertrophic cardiomyopathy. We are able to identify accurately individuals at low risk of sudden death (absence of family history of sudden death, of history of recurrent syncope, absence of frequent non-sustained ventricular tachycardia, and importantly in adolescents and young adults, normal exercise blood pressure response). For the individual who has already suffered an episode of out of hospital cardiac arrest, the decision to implant an automatic cardioverter-defibrillator is usually fairly clear. Does the current evidence justify the routine use of amiodarone with its attendant side effect profile to "high risk" patients, many of whom are young? The answer will only be resolved definitively with an appropriately designed, randomised trial of amiodarone $v$ defibrillator, which is long overdue. This would overcome many of the ethical issues and provide data as to the efficacy of each of these strategies. In the interim, very recent experience of McKenna's group provides further evidence of the beneficial effects of amiodarone in high risk groups. ${ }^{31}$ They stratified 474 consecutive patients according to presence of $\leqslant 1$ risk factor (low risk, $\mathrm{n}=284$ ) or $\geqslant 2$ risk factors (high risk, $\mathrm{n}=109$ ). Mean follow up was 1121 days, and 81 patients received amiodarone. In the group not receiving amiodarone the annual rate of sudden death was $2.5 \%$ in the high risk and $1.3 \%$ in the low risk group; there were no deaths in the treated group. Therefore, although unequivo- cal evidence in support of amiodarone is lacking, the weight of evidence strongly favours the use of amiodarone in high risk groups.

K PRASAD

M P FRENNEAUX

Department of Cardiology,

University of Wales College of Medicine,

Heath Park, Cardiff CF4 $4 X N$, UK

1 Wellens HJ, de Vreede J, Gorgels AP. Sudden cardiac death. How to reduce the number of victims? Eur Heart $\mathcal{F}$ 1995;16(suppl G):7-9

2 Maron BJ. Hypertrophic cardiomyopathy. Curr Probl Cardiol 1993;18:639704 .

3 Doval HC, Nul DR, Grancelli HO, et al. Nonsustained ventricular tachycardia in severe heart failure. Independent marker of increased mortality due to sudden death. GESICA-GEMA Investigators. Circulation 1996;94: 3198-203.

4 A randomized trial of propranolol in patients with acute myocardial infarction. I. Mortality results. $¥ A M A 1982 ; 247: 1707-14$

5 The Cardiac Arrhythmia Suppression Trial (CAST) Investigators. Preliminary report: effect of encainide and flecainide on mortality in a randomized nary report: effect of encainide and flecainide on mortality in a randomized
trial of arrhythmia suppression after myocardial infarction. $N$ Engl $\mathcal{F}$ Med 1989;321:406-12.

6 Waldo AL, Camm AJ, deRuyter H, et al. Effect of d-sotalol on mortality in patients with left ventricular dysfunction after recent and remote myocardial infarction. The SWORD Investigators. Survival with oral d-sotalol. Lancet 1996;348:7-12.

Singh BN. Antiarrhythmic actions of amiodarone: a profile of a paradoxical agent. Am $\mathcal{F}$ Cardiol 1996;78:41-53.

8 Doval HC, Nul DR, Grancelli HO, et al. Randomized trial of low-dose amiodarone in severe congestive heart failure. Grupo de Estudio de la Sobrevida en la Insuficiencia Cardiaca en Argentina (GESICA). Lancet 1994;344:493-8.

9 Singh SN, Fletcher RD, Fisher SG, et al. Amiodarone in patients with congestive heart failure and asymptomatic ventricular arrhythmia. Survival trial of antiarrhythmic therapy in congestive heart failure. $N$ Engl $\mathcal{F}$ Med 1995;333:77-82

10 Cairns JA, Connolly SJ, Roberts R, et al. Randomized trial of outcome after myocardial infarction in patients with frequent or repetitive ventricular premature depolarisations: CAMIAT. Canadian Amiodarone Myocardial Infmature depolarisations: CAMIAT. Canadian Amiodarone Myocard

11 Julian DG, Camm AJ, Frangin G, et al. Randomized trial of effect of amiodarone on mortality in patients with left-ventricular dysfunction after recent myocardial infarction: EMIAT. European Myocardial Infarct Amiodarone Trial Investigators. Lancet 1997;349:667-74

12 McKenna WJ, Camm AJ. Sudden death in hypertrophic cardiomyopathy. Assessment of patients at high risk. Circulation 1989;80:1489-92.

13 McKenna WJ, Deanfield J, Faruqui A, et al. Prognosis in hypertrophic cardiomyopathy: role of age and clinical, electrocardiographic and hemodynamic features. Am f Cardiol 1981;47:532-8.

14 Spirito P, Seidman CE, McKenna WJ, et al. The management of hypertrophic cardiomyopathy. N Engl f Med 1997;336:775-85.

15 Sadoul N, Prasad K, Elliott PM, et al. Prospective assessment of blood pressure response as a marker of risk of sudden death in hypertrophic cardiomyopathy. Circulation 1997;96:2987-91.

16 Maron BJ, Savage DD, Wolfson JK, et al. Prognostic significance of 24 hour ambulatory electrocardiographic monitoring in patients with hypertrophic cardiomyopathy: a prospective study. Am f Cardiol 1981;48:252-7.

17 McKenna WJ, England D, Doi YL, et al. Arrhythmia in hypertrophic McKenna WJ, England D, Doi YL, et al. Arrhythmia in hypertrop
cardiomyopathy. I: influence on prognosis. Br Heart f 1981;46:168-72.

18 McKenna WJ, Oakley CM, Krikler DM, et al. Improved survival with amiodarone in patients with hypertrophic cardiomyopathy and ventricular darone in patients with hypertrophic
tachycardia. Br Heart $\mathcal{f} 1985 ; 53: 412-16$.

19 McKenna WJ, Franklin RC, Nihoyannopoulos P, et al. Arrhythmia and prognosis in infants, children and adolescents with hypertrophic cardiomyopathy. I Am Coll Cardiol 1988;11:147-53.

20 Spirito $\mathrm{P}$, Chiarella F, Carratino L, et al. Clinical course and prognosis of hypertrophic cardiomyopathy in an outpatient population. $N$ Engl f Med 1989;320:749-55.

21 Maron BJ, Spirito P. Impact of patient selection biases on the perception of hypertrophic cardiomyopathy and its natural history. $\mathrm{Am} \mathrm{f}$ Cardiol 1993;72:970-2.

22 Kofflard MJ, Waldstein DJ, Vos J, et al. Prognosis in hypertrophic cardiomyopathy observed in a large clinic population. Am f Cardiol 1993;72:939-43.

23 Bosson C, Hagège A, Desnos $M$, et al. Prognosis in hypertrophic cardiomyopathy: study of a large French population [abstract]. Eur Heart $\mathcal{f}$ 1996;17(suppl):p507.

24 Spirito P, Rapezzi C, Autore C, et al. Prognosis of asymptomatic patients with hypertrophic cardiomyopathy and non-sustained ventricular tachycarwith hypertrophic cardiomyopath
dia. Circulation 1994;90:2743-7.

25 Gilligan DM, Missouris CG, Boyd MJ, et al. Sudden death due to ventricular tachycardia during amiodarone therapy in familial hypertrophic cardiomyopathy. Am f Cardiol 1991;68:971-3.

26 Fananapazir L, Leon MB, Bonow RO, et al. Sudden death during empiric amiodarone therapy in symptomatic hypertrophic cardiomyopathy. Am $\mathcal{F}$ Cardiol 1991;67:169-74.

27 Fananapazir L, Epstein SE. Value of electrophysiologic studies in hypertrophic cardiomyopathy treated with amiodarone. Am f Cardiol 1991; 67:175-82.

28 Mercereau D, Kubac G, Klinke WP. Failure of amiodarone to prevent ventricular fibrillation (sudden death) in hypertrophic cardiomyopathy. Can $\mathcal{F}$ Cardiol 1989;5:77-80.

29 Haffajee CI, Love JC, Alpert JS, et al. Efficacy and safety of long-term amiodarone in treatment of cardiac arrhythmias: dosage experience. Am Heart $\mathcal{F}$ 1983;106:935-43.

30 Cecchi F, Olivotto I, Montereggi A, et al. Prognostic value of non-sustained ventricular tachycardia and the potential role of amiodarone therapy in ventricular tachycardia and the potential role of amiodarone therapy in hypertrophic cardiomyopathy: assessme
based population. Heart 1998;79:331-6.

31 Elliott PM, Sharma S, Poloniecki J, et al. Amiodarone and sudden death in hypertrophic cardiomyopathy [abstract]. Circulation 1997;96(suppl):I-464. 